J. Lake Sci. (湖泊科学), 2017, 29(3): 575-584

DOI 10. 18307/2017. 0306

(c) 2017 by Journal of Lake Sciences

\title{
利用不同物候期水生植物配置提高浮床人工湿地的除氮效果
}

\author{
张 惠 ${ }^{1}$, 汪鹏合 ${ }^{1}$, 张文娟 ${ }^{1}$, 左 杰 $^{1}$, 邹祥旭 ${ }^{1}$, 季 军 $^{2}$, 安树青 ${ }^{1,3}$, 赵德华 ${ }^{1 * *}$ \\ ( 1 : 南京大学生命科学学院, 南京 210046) \\ (2: 洪泽县清盈尾水湿地管理有限公司, 淮安 223001) \\ (3: 南京大学常熟生态研究院, 常熟 215500)
}

摘 要: 为验证利用不同物候期水生植物配置提高碳氮比失衡湿地脱氮能力的可行性, 设计了向水芹菜 (Oenanthe javani$c a$ ) 浮床人工湿地系统中添加轮叶黑藻 (Hydrilla verticillata) 的实验, 研究了在秋、冬季低温条件下, 暖季型植物分解释碳 对系统氮去除效果的影响. 结果表明, 添加轮叶黑藻显著提高了系统水体碳氮比, 促进反硝化作用, 提高总氮和硝态氮的 去除率. 在 4 个周期中, 与对照组相比, 物候期组合型浮床人工湿地系统的总氮去除率分别提升 $23.03 \% 、 10.90 \% 、 18.55 \%$ 和 $22.93 \%$, 硝态氮去除率分别提升 $38.28 \% 、 20.74 \% 、 17.87 \%$ 和 $17.06 \%$. 由此可见, 利用暖季型和冷季型植物配置提高碳 氮比失衡湿地氮去除率是可行的.

关键词: 浮床人工湿地;水芹菜; 轮叶黑藻;碳氮比;氮去除

\section{Improving nitrogen removal by phenophase arrangement of aquatic macrophyte species in the floating-bed constructed wetland}

ZHANG Hui $^{1}$, WANG Penghe ${ }^{1}$, ZHANG Wenjuan ${ }^{1}$, ZUO Jie ${ }^{1}$, ZOU Xiangxu ${ }^{1}$, JI Jun ${ }^{2}$, AN Shuqing ${ }^{1,3}$ \& ZHAO Dehua ${ }^{1 * *}$

(1: School of Life Science, Nanjing University, Nanjing 210046, P.R.China)

(2: Qingying Tail Water Wetland Management Limited Company of Hongze County, Huaian 223001, P.R.China)

(3: Ecology Research Institute of Changshu, Nanjing University, Changshu 215500, P.R. China)

Abstract: The main objective of this study is to identify the feasibility of improving the nitrogen removal efficiency in low $\mathrm{C} / \mathrm{N}$ wetlands by arranging aquatic macrophyte species with different phonological characteristics. We conducted a pilot experiment, adding warm-season species of Hydrilla verticillata to cold-season species of Oenanthe javanica at a floating-bed constructed wetland system $(\mathrm{FCW})$, in order to quantify the effects of the decomposition of the warm-season macrophyte residue on the nitrogen removal efficiency under the condition of low temperature during autumn and winter seasons. The results showed that the adding of $H$. verticillata significantly increased the $\mathrm{C} / \mathrm{N}$ ratios in the organic carbon shortage systems of $\mathrm{FCW}$, and thus improved total nitrogen and nitrate nitrogen removal efficiency due to stimulating denitrification activities. During the four cycle periods of the phenophase-combined FCW, compared with the control group, the removal efficiency of total nitrogen in each period was increased by $23.03 \%$, $10.90 \%, 18.55 \%$ and $22.93 \%$ respectively, and the removal efficiency of nitrate nitrogen was increased by $38.28 \%, 20.74 \%$, $17.87 \%$ and $17.06 \%$, respectively. Therefore we conclude that it is feasible to improve the nitrogen removal efficiency by re-arrangement of warm and cool season plants in the low $\mathrm{C} / \mathrm{N}$ wetlands.

Keywords: Floating-bed constructed wetland; Oenanthe javanica; Hydrilla verticillata; $\mathrm{C} / \mathrm{N}$ ratios; nitrogen removal

人工湿地是一种利用基质、水体、植物及微生物的物理、化学、生物三重协同作用实现污水高效净化的 系统 ${ }^{[1]}$, 以其廉价性、高效性、生态化、可以因地制宜地构建及易于分散化处理等优点, 被广泛应用于国内外

* 国家水体污染控制与治理科技重大专项 (2014ZX07204-002) 资助. 2016-06-14 收稿;2016-09-08 收修改稿. 张 惠 (1991 ) ,女, 硕士研究生;E-mail: tofurther@163.com.

** 通信作者;E-mail: dhzhao@ nju.edu.cn. 
各类污、废水的处理 ${ }^{[2-5]}$. 然而, 在实际运行过程中, 人工湿地的氮去除率差异很大, 从 $30 \% \sim 90 \%$ 以上不 等 ${ }^{[6]}$. 在以脱氮为主要目的的人工湿地中, 碳源不足往往构成湿地脱氮的主要限制性因素 ${ }^{[7-8]}$.

研究表明添加碳源可以有效提高人工湿地的脱氮效率 ${ }^{[7,9]}$; 而植物作为一种天然纤维素原料, 因其低成 本、可再生和可用性广泛等特点, 成为外加碳源的良好选择 ${ }^{[10]}$. Ingersoll 等 ${ }^{[11]}$ 以切碎后的香蒲作为反硝化碳 源, 发现碳源添加系统的脱氮效果是对照系统的 10 倍. 对垂直流人工湿地小试模型的研究也表明植物秸秆 添加使得系统总氮 $(\mathrm{TN})$ 去除率从 $44 \%$ 左右提高到了 $53 \% \sim 66 \%{ }^{[12]}$. Shao 等 ${ }^{[13]}$ 和孙雅丽等 ${ }^{[14]}$ 也报道了以植 物生物质作为反硝化碳源对潜流湿地系统硝酸盐去除的促进作用.

但是, 植物碳源的效果受添加方式、添加量和添加时机的影响极大, 已有研究多针对潜流湿地系统, 且 大多使用预处理的植物作为碳源, 这不但增加了成本, 同时也提高了额外的环境风险 ${ }^{[9,15]}$. 对于低污染负 荷、碳氮比 $(\mathrm{C} / \mathrm{N})$ 失衡的湿地而言, 对系统的植物群落进行优化配置可能是一种更好的解决方案: 利用冷季 型植物在春、夏季和暖季型植物在秋、冬季的调亡分解及碳源释放, 调节系统水体的 $\mathrm{C} / \mathrm{N}$ 比, 进而克服系统 氮去除率低的问题. 如被证实可行, 这将是一种更为生态化的解决方案. 尽管目前在植物碳源添加对人工湿 地净化效率的影响和自然条件下植物材料的分解过程两个方面已有大量的研究报道 ${ }^{[16-20]}$, 但是鲜有研究将 这两个方面结合起来, 利用不同植物物候期的交替构建物候期组合型浮床湿地系统进行污水净化的研究尚 未见报道.

因此, 本研究设计了暖季型植物轮叶黑藻 (Hydrilla verticillata) 和冷季型植物水芹菜 (Oenanthe javanica) 组合配置的浮床人工湿地系统, 研究了轮叶黑藻在秋、冬季调亡分解过程中对系统除氮效果的影响, 旨在验 证物候期组合型浮床人工湿地系统提升 $\mathrm{C} / \mathrm{N}$ 比失衡水体氮去除率的可行性, 为人工湿地生态净化工程提供 理论指导.

\section{1 材料与方法}

\section{1 实验装置}

以白色塑料长桶 (长 $\times$ 宽 $\times$ 高 $=1.9 \mathrm{~m} \times 1.1 \mathrm{~m} \times 0.6 \mathrm{~m}$ ) 作为容器模拟表面流人工湿地单元 (图 1 ), 设置 4 个 处理组 ( $\mathrm{LN}$ 组: 贫营养水体+水芹菜; $\mathrm{LA}$ 组: 贫营养水体+水芹菜+轮叶黑藻、 $\mathrm{HN}$ 组: 富营养水体+水芹菜、

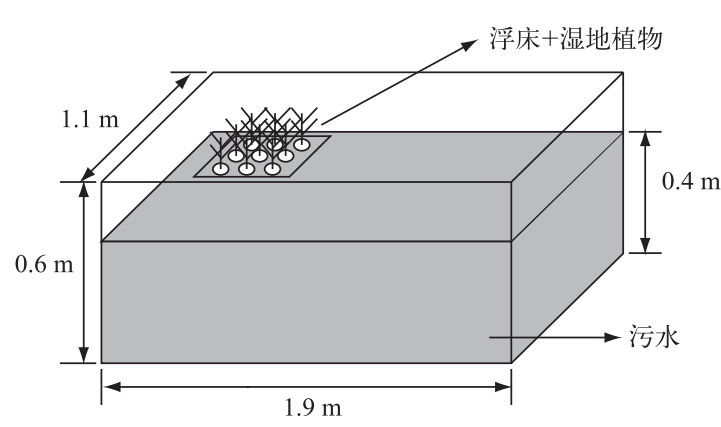

图 1 浮床人工湿地实验装置示意图

Fig.1 Schematic diagram of the experimental model of floating-bed constructed wetland

HA 组: 富营养水体+水芹菜+轮叶黑藻), 每处理 组含 3 个重复单元, 共 12 个浮床人工湿地系统单 元. 以 HCFC500 生态浮床 $(500 \mathrm{~mm} \times 500 \mathrm{~mm} \times 65$ $\mathrm{mm}$, 每个浮床有 9 个栽植孔, 孔径 $8 \mathrm{~cm}$ ) 作为水芹 的生长载体, 每个湿地系统 6 块浮床共 54 筐浮床 植物, 占整个湿地单元表面积的 $72 \%$, 每个系统每 筐水芹的初始生长状况和生物量基本一致, 各单 元水位设定为 $0.4 \mathrm{~m}$. 物候期组合型系统中轮叶黑 藻栽植于小花盆中 (内口直径 $27.9 \mathrm{~cm}$, 底部直径 $18.5 \mathrm{~cm}$, 高 $20.9 \mathrm{~cm}$, 内部填充 $10 \mathrm{~cm}$ 高的细沙以 固定轮叶黑藻), 每个系统 10 盆, 均匀分布在湿地 单元中, 平均每盆轮叶黑藻干重约 $38.17 \sim 38.33$ $\mathrm{g}$, 每个系统总生物量为 $381.73 \sim 383.34 \mathrm{~g}$ (干重).

\section{2 实验材料}

本实验所用的浮床植物水芹菜为一种国内常见的冷季型水生植物, 具有耐低温、高生物量、可重复收割 等特点, 适合浮床人工湿地. 作为暖季型植物的轮叶黑藻取自附近水塘, 经过清水净化培养 $15 \mathrm{~d}$ 后移栽至小 花盆, 再移人人工湿地单元.

\section{3 实验运行状况}

实验于 2015 年 10 月 24 日开始, 12 月 22 日结束, 地点位于南京大学洪泽湿地实验基地 $\left(33^{\circ} 19^{\prime} \mathrm{N}\right.$, $\left.118^{\circ} 55^{\prime} \mathrm{E}\right)$, 以 $15 \mathrm{~d}$ 为一个处理周期, 系统共运行 4 个周期. 以天然池塘水为基础添加药品 $\mathrm{KNO}_{3}$ 和 
$\left(\mathrm{NH}_{4}\right)_{2} \mathrm{SO}_{4}$ 配制成富营养水体, 其中 $\mathrm{KNO}_{3}$ 每周期添加 $5 \mathrm{mg} / \mathrm{L},\left(\mathrm{NH}_{4}\right)_{2} \mathrm{SO}_{4}$ 每周期添加 $10 \mathrm{mg} / \mathrm{L}$ (每周期配制 一次, 上一周期剩余的营养物质累加到下一周期), 4 个周期 $\mathrm{TN}$ 、硝态氮 $\left(\mathrm{NO}_{3}^{-}-\mathrm{N}\right)$ 和铵态氮 $\left(\mathrm{NH}_{4}^{+}-\mathrm{N}\right)$ 初始浓 度分别在 $13.73 \sim 33.68 、 2.13 \sim 6.08$ 和 7.97 9.09 $\mathrm{mg} / \mathrm{L}$ 之间. 不添加药品的池塘水作为贫营养水体 (水质指 标 $\mathrm{TN} 、 \mathrm{NO}_{3}^{-}-\mathrm{N} 、 \mathrm{NH}_{4}^{+}-\mathrm{N}$ 的初始浓度分别为 $1.27 、 0.28$ 和 $\left.0.74 \mathrm{mg} / \mathrm{L}\right)$.

实验运行期间,水体 $\mathrm{pH}$ 值在 $8.09 \sim 10.45$ 之间波 动, 水温逐渐降低 (图 2). 在 10 月 24 日-11月 23 日 期间, 水温从 $21^{\circ} \mathrm{C}$ 逐渐降至 $10^{\circ} \mathrm{C} ; 11$ 月 28 日迅速降 至 $5^{\circ} \mathrm{C}$ 以下, 之后一直在 $5^{\circ} \mathrm{C}$ 左右波动.

\section{4 主要监测指标及方法}

每个周期结束时于水下 $20 \mathrm{~cm}$ 处取水样, 按照标 准操作程序利用哈希 $\mathrm{DR} / 2800$ 分光光度计对化学需 氧量 ( $\mathrm{COD}) 、 \mathrm{TN} 、 \mathrm{NO}_{3}^{-}-\mathrm{N} 、 \mathrm{NH}_{4}^{+}-\mathrm{N}$ 浓度进行测定, 其中, 依据美国国家环境保护局 (USEPA) 标准: COD 浓度 测定采用 USEPA 消解比色法, TN 浓度测定采用过硫 酸盐氧化法, $\mathrm{NO}_{3}^{-}-\mathrm{N}$ 浓度测定采用镉还原法, $\mathrm{NH}_{4}^{+}-\mathrm{N}$ 浓度测定采用水杨酸法; 使用美国哈希 HQ40d 双路 输人多参数数字分析仪检测水体溶解氧 (DO) 浓度及

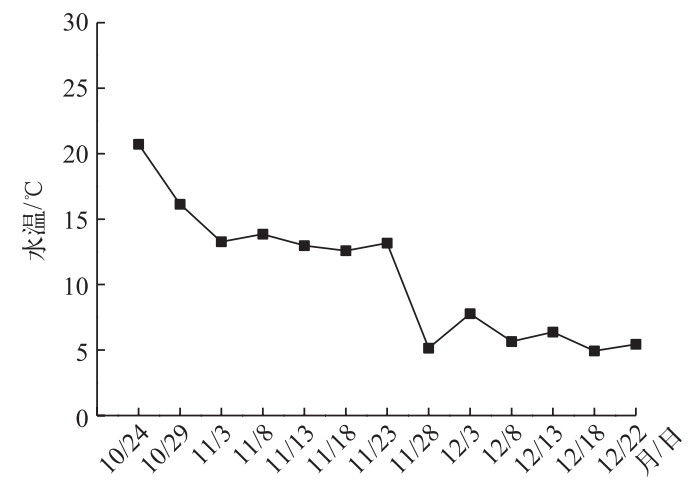

图 2 水温随时间的变化

Fig. 2 Changes of water temprature over time $\mathrm{pH}$; 并在水体底部安装 HOBO UA-002-64 防水型温度光照记录仪监测系统水温变化.

同时, 每个系统取 3 筐水芹菜, 采用氯化三苯基四氮唑 (TTC) 法 ${ }^{[21]}$ 检测湿地植物根系活力, 并及时补充 水芹菜以保持植株密度不变; 组合型系统每个系统取 1 盆轮叶黑藻, 洗净后烘干称重估算组合型系统中轮 叶黑藻生物量变化.

\section{5 数据处理}

实验数据采用 SPSS 17.0 统计软件进行 One-way ANOVA 分析, 显著性水平设为 0.05 , 采用 OriginPro 8.0 软件作图.

\section{2 结果与分析}

\section{1 暖季型植物分解及碳源溶出过程}

2.1.1 轮叶黑藻分解过程中生物量的变化 根据轮叶黑藻生物量的变化, 可以将其调亡分解过程分为两个阶 段: 前两个周期干重迅速下降, 为快速分解期, LA 、 HA 处理组分别下降 181.94 和 $182.08 \mathrm{~g}$; 第 3、4 周期, 轮叶 黑藻干重减少缓慢, 为缓慢分解期, LA 、 HA 两组分别下降 36.78 和 $35.53 \mathrm{~g}$ (图 3a). 实验结束后, 轮叶黑藻剩 余干重为初始时的 $43 \%$, 分解掉超过自身一半的物质. 这表明在实验前期温度较高, 轮叶黑藻分解迅速, 可 为湿地系统提供较多的碳源, 而随着温度的降低, 分解减慢, 提供碳源能力下降.

2.1.2 水体 COD 浓度及 $\mathrm{C} / \mathrm{N}$ 比随时间的变化 各处理组水体 COD 初始浓度基本一致, 平均为 $16.63 \mathrm{mg} / \mathrm{L}$. 实验期间, LN 组的水体 COD 值略有上升, 实验结束时为 $20.33 \mathrm{mg} / \mathrm{L} ; \mathrm{LA} 、 \mathrm{HN} 、 \mathrm{HA}$ 组的水体 COD 浓度上升明 显, 实验结束时, 3 组处理分别达到 38.83、31.46 和 $36.04 \mathrm{mg} / \mathrm{L}$. 每个周期 LA、HA 组水体的 COD 浓度均分别 高于 LN、HN 组 (图 3b), 对实验数据进行单因素方差分析, 在前 3 个周期中, LA、LN、HA 与 HN 组两两之间 $\mathrm{COD}$ 浓度均差异极显著 $(P<0.01)$, 在第 4 周期时均差异显著 $(P<0.05)$.

实验期间, $\mathrm{LN}$ 与 $\mathrm{LA}$ 组水体平均 $\mathrm{C} / \mathrm{N}$ 比除第 3 周期外均存在极显著差异 $(P<0.01)$, 且 $\mathrm{LA}$ 组是 $\mathrm{LN}$ 组的 $1.35 \sim 4.32$ 倍. $\mathrm{HN}$ 与 $\mathrm{HA}$ 组水体平均 $\mathrm{C} / \mathrm{N}$ 比 4 个周期均存在极显著差异 $(P<0.01), \mathrm{HA}$ 组是 $\mathrm{HN}$ 组的 $1.68 \sim$ 2.36 倍 (表 1). 表明轮叶黑藻分解达到了提高系统内 COD 浓度和改善 $\mathrm{C} / \mathrm{N}$ 比的预期目标, 但其改善作用在 实验进行 1 个月后迅速下降.

2.1.3 LN 与 $\mathrm{LA}$ 组水体氮浓度随时间的变化 实验过程中, $\mathrm{LN}$ 与 $\mathrm{LA}$ 组水体的 $\mathrm{TN} 、 \mathrm{NH}_{4}^{+}-\mathrm{N}$ 浓度具有相似的 变化趋势和过程 (图 4a、4c), 第 1 周期迅速下降, 其中 LN、LA 组 TN 浓度分别下降至 0.3 和 $0.1 \mathrm{mg} / \mathrm{L}$, 差异 显著 $(P<0.05), \mathrm{NH}_{4}^{+}-\mathrm{N}$ 浓度分别下降至 0.067 和 $0.013 \mathrm{mg} / \mathrm{L}$, 差异不显著 $(P>0.05)$ (图 $4 \mathrm{c}$ ). 之后两处理组 

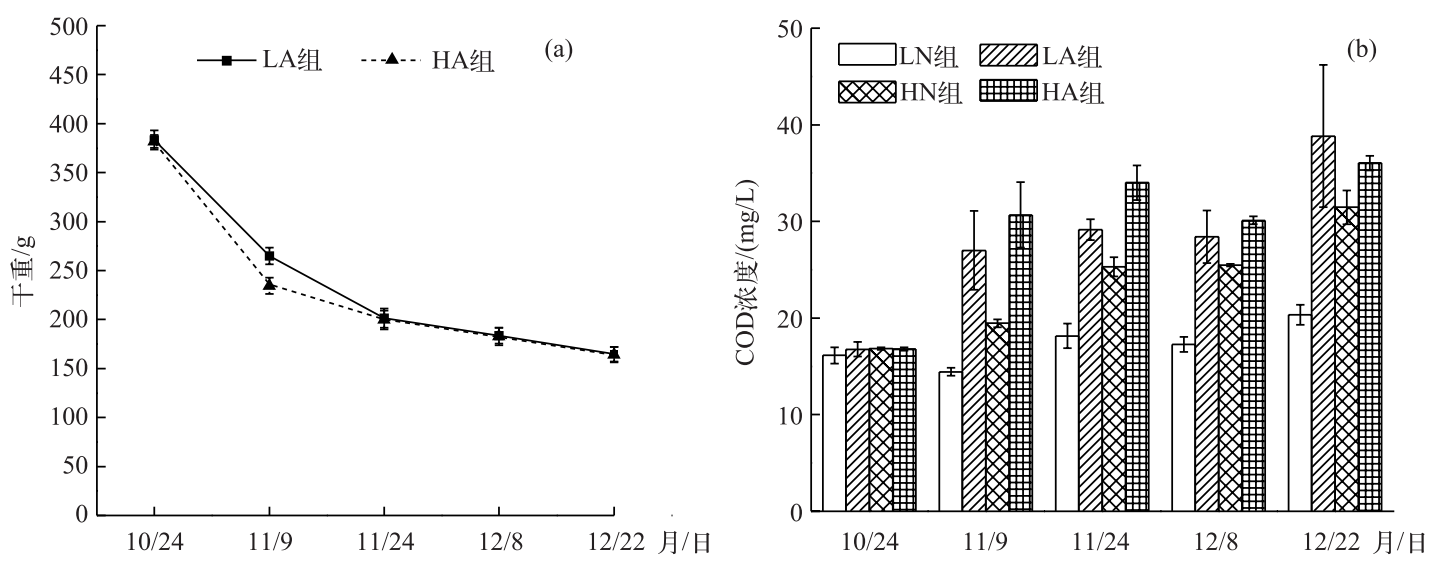

图 3 轮叶黑藻剩余干重 $(a)$ 及水体 COD 浓度 $(b)$ 的变化

Fig.3 Changes of residual dry biomass of Hydrilla verticillata (a) and COD concentration of water (b)

表 1 各周期 LN、LA、HN、HA 组水体平均 $\mathrm{C} / \mathrm{N}$ 比

Tab.1 Average $\mathrm{C} / \mathrm{N}$ ratio of water in $\mathrm{LN}, \mathrm{LA}, \mathrm{HN}$ and $\mathrm{HA}$ groups during each cycle

\begin{tabular}{ccccc}
\hline \multirow{2}{*}{ 周期 } & \multicolumn{4}{c}{$\mathrm{C} / \mathrm{N}$ 比 } \\
\cline { 2 - 5 } & $\mathrm{LN}$ 组 & LA 组 & HN 组 & HA 组 \\
\hline 1 & $32.72 \pm 10.19$ & $141.49 \pm 25.14^{* *}$ & $2.07 \pm 0.37$ & $4.88 \pm 0.69^{* *}$ \\
2 & $61.38 \pm 18.23$ & $172.75 \pm 17.1^{* *}$ & $1.56 \pm 0.16$ & $3.00 \pm 0.15^{* *}$ \\
3 & $73.96 \pm 18.12$ & $99.83 \pm 14.25$ & $1.12 \pm 0.03$ & $1.88 \pm 0.10^{* *}$ \\
4 & $89.50 \pm 11.67$ & $161.09 \pm 19.62^{* * *}$ & $0.87 \pm 0.05$ & $1.52 \pm 0.07^{* *}$ \\
\hline
\end{tabular}

** 分别表示同一周期的 $\mathrm{LN}$ 与 $\mathrm{LA}$ 组、HN 与 $\mathrm{HA}$ 组水体 $\mathrm{C} / \mathrm{N}$ 比差异极显著 $(P<0.01, n=3)$.

的 $\mathrm{TN} 、 \mathrm{NH}_{4}^{+}-\mathrm{N}$ 浓度波动较小, 维持在较低水平, 且差异不显著 $(P>0.05)$; 两处理组的 $\mathrm{NO}_{3}^{-}-\mathrm{N}$ 浓度在第 1 周期 也迅速下降, 分别达 0.03 和 $0 \mathrm{mg} / \mathrm{L}$, 之后都在 $0.1 \mathrm{mg} / \mathrm{L}$ 上下波动 (图 $4 \mathrm{~b}$ ), 4 个周期两处理组的 $\mathrm{NO}_{3}^{-}-\mathrm{N}$ 浓度 均无显著差异 $(P>0.05)$. 这说明轮叶黑藻的分解未引起系统水体氮浓度的显著升高.

\section{$2.2 \mathrm{HN}$ 和 $\mathrm{HA}$ 组水体 $\mathrm{TN}, \mathrm{NO}_{3}^{-}-\mathrm{N}$ 和 $\mathrm{NH}_{4}^{+}-\mathrm{N}$ 的去除率}

在实验进行的 4 个周期中, $\mathrm{HN}$ 组的 $\mathrm{TN}$ 去除率分别为 50.61\%、36.49\%、12.53\% 和 5.64\%, HA 组分别为 $73.64 \% 、 47.39 \% 、 31.08 \%$ 和 $28.57 \%$ ( 图 5a). 各个周期 HA 组的 TN 去除率均极显著高于 $\mathrm{HN}$ 组 $(P<0.01$ ).

$\mathrm{HN}$ 组的 $\mathrm{NO}_{3}^{-}-\mathrm{N}$ 去除率分别为 $18.34 \% 、 46.76 \% 、 20.71 \%$ 和 $11.53 \%, \mathrm{HA}$ 组分别为 $56.62 \% 、 67.5 \%$ 、 $38.58 \%$ 和 $28.59 \%$ （图 5b)，每个周期 $\mathrm{HA}$ 组的 $\mathrm{NO}_{3}^{-}-\mathrm{N}$ 去除率均极显著高于 $\mathrm{HN}$ 组 $(P<0.01)$.

$\mathrm{HN} 、 \mathrm{HA}$ 两处理组每个周期的 $\mathrm{NH}_{4}^{+}-\mathrm{N}$ 去除率均高于 $80 \%$ (图 5c), HA 组比 $\mathrm{HN}$ 组的去除优势更明显, 除 第 2 周期外,两处理组的 $\mathrm{NH}_{4}^{+}-\mathrm{N}$ 去除率均存在显著差异 $(P<0.05)$.

与 $\mathrm{HN}$ 组相比, $\mathrm{HA}$ 组 4 个实验周期的 $\mathrm{TN} 、 \mathrm{NO}_{3}^{-}-\mathrm{N}$ 和 $\mathrm{NH}_{4}^{+}-\mathrm{N}$ 去除率平均高出 $18.85 \% 、 23.49 \%$ 和 $4.69 \%$. 表明轮叶黑藻分解可改善低 $\mathrm{C} / \mathrm{N}$ 比湿地碳供应, 并主要通过促进系统的 $\mathrm{NO}_{3}^{-}-\mathrm{N}$ 去除进而提高系统的氮去 除率.

\section{3 不同处理组水体溶解氧浓度和浮床植物根系活力的变化}

LA、HA 组水体溶解氧浓度在第 1 周期显著下降 $(P<0.01), \mathrm{LN} 、 \mathrm{HN}$ 组基本无变化( 图 6a). 第 $1 、 2$ 周期 结束时, LA 组水体溶解氧浓度分别为 4.21 和 $6.43 \mathrm{mg} / \mathrm{L}$, 显著低于 $\mathrm{LN}$ 组 (分别为 7.99 和 $10.53 \mathrm{mg} / \mathrm{L}$ ) ; 同 样, HA 组在 $1 、 2$ 周期结束时水体溶解氧浓度分别为 4.61 和 $7.08 \mathrm{mg} / \mathrm{L}$, 显著低于 $\mathrm{HN}$ 组(分别为 9.58 和 8.77 $\mathrm{mg} / \mathrm{L}$ ). 后期各个处理组的水体溶解氧浓度均上升, 实验结束时 (12 月 22 日) LN、LA、HN、HA 4 个处理组的 

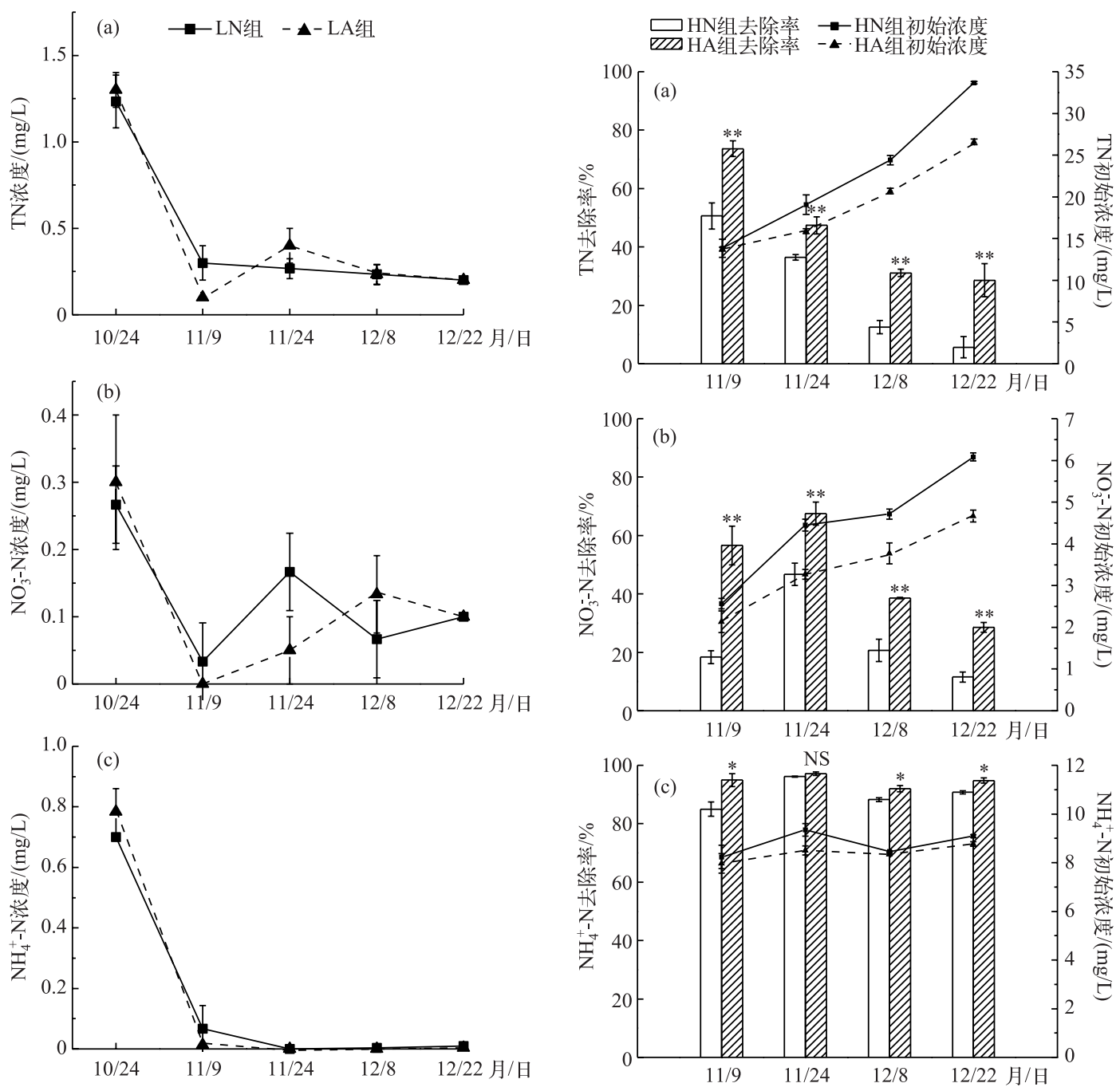

图 4 各周期 $\mathrm{LN}$ 和 $\mathrm{LA}$ 组中 $\mathrm{TN}(\mathrm{a}) 、 \mathrm{NO}_{3}^{-}-\mathrm{N}(\mathrm{b})$ 和 $\mathrm{NH}_{4}^{+}-\mathrm{N}(\mathrm{c})$ 浓度变化

Fig.4 Changes of $\mathrm{TN}(\mathrm{a}), \mathrm{NO}_{3}^{-}-\mathrm{N}(\mathrm{b})$ and $\mathrm{NH}_{4}^{+}-\mathrm{N}$ (c) concentrations in $\mathrm{LN}$ and $\mathrm{LA}$ groups during each cycle

图 5 各周期 $\mathrm{HN}$ 和 $\mathrm{HA}$ 组中 $\mathrm{TN}(\mathrm{a}) 、 \mathrm{NO}_{3}^{-}-\mathrm{N}(\mathrm{b}) 、$ $\mathrm{NH}_{4}^{+}-\mathrm{N}$ (c) 的初始浓度和平均去除率(同一周期 **表示差异极显著 $(P<0.01), *$ 表示差异显著 $(P<0.05), \mathrm{NS}$ 表示差异不显著 $(n=3))$

Fig.5 Initial concentrations and average removal efficiencies of $\mathrm{TN}(\mathrm{a}), \mathrm{NO}_{3}^{-}-\mathrm{N}(\mathrm{b})$ and $\mathrm{NH}_{4}^{+}-\mathrm{N}(\mathrm{c})$ in $\mathrm{HN}$ and $\mathrm{HA}$ groups during each cycle

水体溶氧量浓度分别为 $13.20 、 12.68 、 12.35$ 和 $12.35 \mathrm{mg} / \mathrm{L}$, 处理组间差异不显著 $(P>0.05)$. 表明在实验前 期, 添加轮叶黑藻可引起系统内溶解氧浓度的下降, 而实验后期随着轮叶黑藻分解速度的下降, 对系统中溶 解氧浓度的影响逐渐减小.

随实验的进行, 各个处理组水芹菜根系活力总体呈下降趋势 (图 6b). 其中, LN 组的根系活力在实验结 束时为 $31.73 \mu \mathrm{g} /(\mathrm{g} \cdot 2 \mathrm{~h})$; LA 组水芹菜根系活力在前两个周期迅速下降, 随后下降速度逐渐减缓, 且每个 周期都低于 $\mathrm{LN}$ 组, 实验结束时为 $26.67 \mu \mathrm{g} /(\mathrm{g} \cdot 2 \mathrm{~h})$; HN 组在第 1 周期较初始值有所上升, 之后逐渐下降, 
实验结束时为 $63.73 \mu \mathrm{g} /(\mathrm{g} \cdot 2 \mathrm{~h})$; HA 组的变化趋势与 LA 组相似, 且每个周期都低于 $\mathrm{HN}$ 组, 实验结束时为 $35.2 \mu \mathrm{g} /(\mathrm{g} \cdot 2 \mathrm{~h})$. 这表明添加轮叶黑藻可引起水芹菜根系活力的下降.

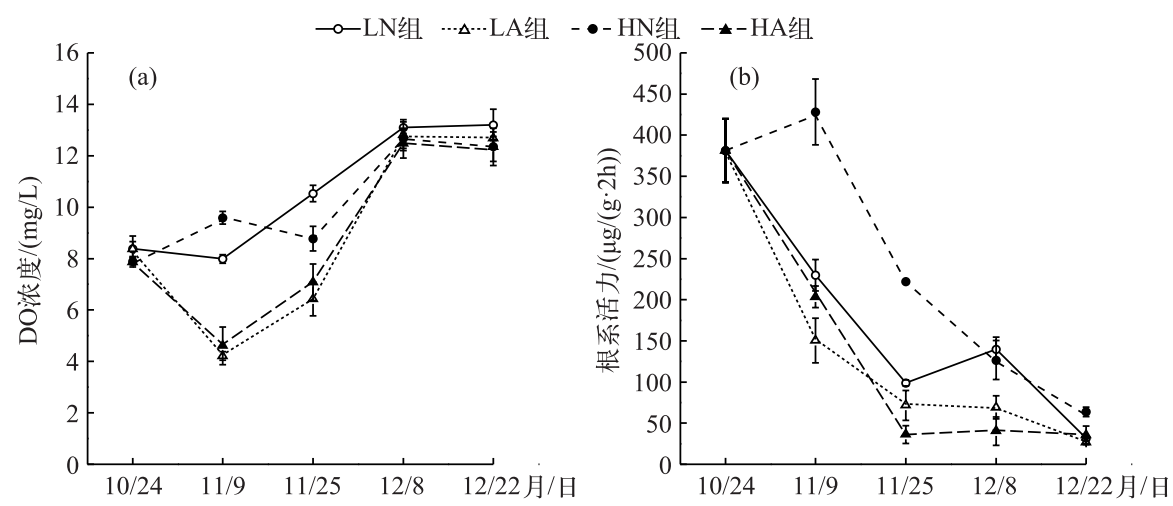

图 6 各周期各处理组水体溶解氧浓度 (a) 和水芹菜根系活力 ( b) 的变化

Fig.6 Changes of dissolved oxygen concentration of water (a) and root activity of

Oenanthe javanica (b) of different groups during each cycle

\section{3 讨论}

植物在调亡过程中, 会释放大量有机物质进人水体, 导致水体有机碳浓度增加. 叶春等 ${ }^{[22]}$ 的室内模拟 研究发现, 黑藻在腐解早期会导致水体总有机碳浓度迅速上升, 整个过程总有机碳释放率为 $81.31 \%$; 甘蔗渣 在固液比 $1: 80$ 水解时, COD 释放量可达 $45.45 \mathrm{mg} / \mathrm{L}^{[18]}$; 谢理等 ${ }^{[23]}$ 研究了成熟期挺水植物荠草和芦苇的叶 在水环境中的降解过程, 指出植物样品干重添加量为 $28.6 \mathrm{~g} / \mathrm{L}$ 时, 分解前期 $(0 \sim 8 \mathrm{~h})$ 荠草和芦苇溶解性有机 碳释放量分别可以达到 1249.8 和 $1364.4 \mathrm{mg} / \mathrm{L}$. 本研究所选用的碳源植物轮叶黑藻喜温、耐热, 其生长期可 以从每年的 3 月持续到 11 月,之后植株开始衰亡、解体 ${ }^{[24]}$. 实验开始时轮叶黑藻刚进人衰亡期, 4 个周期内 物候期组合型系统的水体 COD 值远高于对照系统, 说明轮叶黑藻在秋、冬季衰亡期的分解能够释放大量的 有机物, 可以作为外加碳源提高系统的有机碳浓度. 对比 LN 与 LA 两处理组水体氮浓度的变化可知, 轮叶黑 藻分解未引起系统水体氮污染.

人工湿地的氮去除途径主要包括植物吸收、氨氮挥发、基质吸附和微生物硝化一反硝化作用等 ${ }^{[25-26]}$, 其 中微生物硝化一反硝化是主要途径 ${ }^{[1,5,27-31]}$, 通常占到系统氮去除总量的 $60 \% \sim 86 \%{ }^{[32]}$. 而有机碳源作为反硝 化作用的电子供体, 是反硝化过程中必不可少的物质 ${ }^{[8-9,33-34]}$, 生物反硝化过程需要足够数量的碳源, 保证一 定的 $\mathrm{C} / \mathrm{N}$ 比才能顺利完成 ${ }^{[30,35-37]}$. 陆松柳等 ${ }^{[38]}$ 的研究发现, 在进水 $\mathrm{NO}_{3}^{-}-\mathrm{N}$ 浓度为 $25 \sim 45 \mathrm{mg} / \mathrm{L}$ 时, 通过投加 碳源使 $\mathrm{C} / \mathrm{N}$ 比达 1.0 1.2, 潜流式人工湿地的冬季 $\mathrm{NO}_{3}^{-}-\mathrm{N}$ 去除率可从 $5 \%$ 提升到 $35 \%$ 以上. 赵联芳等 ${ }^{[39]}$ 将 芦苇笔添加到垂直流人工湿地表面, 芦苇竿添加量为 $1.0 \mathrm{~kg} / \mathrm{m}^{2}$ 时, TN 去除率由未添加时的 $60 \%$ 提高到 $80 \%$. Wen 等 ${ }^{[10]}$ 研究潜流人工湿地中植物生物质对硝态氮去除的影响发现, 植物碳源添加组的硝酸盐去除 率是非添加组的 2.4 3.0 倍. 可见, 碳源供应对湿地反硝化过程具有很大影响. 在当前研究中, HA 组各周期 水体平均 $\mathrm{C} / \mathrm{N}$ 比均高于 $\mathrm{HN}$ 组, 后期 $\mathrm{HA}$ 组的 $\mathrm{C} / \mathrm{N}$ 比下降, 可能是由于轮叶黑藻投加量不足和温度太低, 轮 叶黑藻分解速度变慢, 导致水体有机碳浓度上升幅度不大. $\mathrm{C} / \mathrm{N}$ 比的差异造成 $\mathrm{HN}$ 和 HA 两处理的脱氮效果 存在显著差异. 可见, 通过轮叶黑藻分解释放碳源, 改善系统 $\mathrm{C} / \mathrm{N}$ 比, 可以促进系统的反硝化作用过程, 提高 系统脱氮效果, 表明不同物候期组合处理是提升秋冬季人工湿地脱氮能力的良好选择. 随着实验的进行, 两 种处理的 $\mathrm{TN}$ 和 $\mathrm{NO}_{3}^{-}-\mathrm{N}$ 去除率都逐渐降低, 可能是因为 $\mathrm{C} / \mathrm{N}$ 比逐渐减小, 温度逐渐降低、微生物活性减弱以 及低温造成水体溶氧量浓度升高, 使反硝化速率降低 ${ }^{[40-41]}$. 后期 $\mathrm{NO}_{3}^{-}-\mathrm{N}$ 去除率的降低也成为 $\mathrm{TN}$ 去除率降 低的原因之一.

同时, 轮叶黑藻在调亡分解过程中消耗了水体的溶解氧, 导致物候期组合型系统的水体溶解氧浓度在 
第 $1 、 2$ 周期显著低于对照系统, 这与张菊等 ${ }^{[42]}$ 的研究结果一致. 水体中溶解氧的多少是衡量水体自净能力 的一个指标. 低溶解氧浓度可以提供还原环境, 能够增加与纤维素、半纤维素降解有关的胞外酶的累积量, 促进植物生物质分解释放有机碳 ${ }^{[43]}$; 有利于含亚硝态氮还原酶细菌及含氮氧化物还原酶细菌的生长, 提高 湿地反硝化速率 ${ }^{[44-45]}$. 而硝化过程是一个需氧过程, 研究表明将 $1 \mathrm{~g}$ 氨氮氧化为硝酸根需消耗 $4.6 \mathrm{~g}$ 氧气, 溶 解氧浓度达到 $1 \mathrm{mg} / \mathrm{L}$ 才能满足氨氧化的需要 ${ }^{[46]}$. 在当前研究中, 物候期组合型系统中水体溶解氧浓度在第 1 周期结束时达到最低值 $4.21 \mathrm{mg} / \mathrm{L}$, 高于硝化反应发生的先决条件, 同时各周期内较高的氨氮去除率也说 明轮叶黑藻分解耗氧未对硝化反应造成不利影响. 但系统水体溶解氧不足会对浮床植物的根部呼吸造成负 面影响, 进而降低植物根系活力, 影响植物生长速率 ${ }^{[47-48]}$. 在当前研究中, 组合型系统中水芹菜的根系活力 普遍更低, 这和轮叶黑藻分解消耗水体溶解氧, 导致水芹菜根系吸收所需能量的供应不足有关. 植物对湿地 净化过程有多方面的影响, 一方面可以通过直接吸收去除水体中的营养物质, 在我们先前的研究中发现, 冬 季水芹菜吸收的氮占 TN 去除的 $15.01 \%^{[49]}$; 另一方面还可以通过为微生物生长提供附着面以及通过根系新 陈代谢和有机物分泌为反硝化微生物提供碳源和能量, 从而影响系统的氮去除效果 ${ }^{[50-51]}$, 有研究指出, 植物 与湿地系统中其他因子的协同作用对湿地 TN 去除率的贡献达 $47 \%{ }^{[52]}$. 因此, 轮叶黑藻分解耗氧降低植物 根系活力也会间接影响植物在湿地净化中的贡献. 在当前研究的后期, 各个系统的水体溶解氧浓度均呈上 升趋势, 这可能与后期轮叶黑藻分解逐渐减弱、耗氧减少 ${ }^{[22]}$ 以及温度降低、水体中氧气的溶解度增加 有关 ${ }^{[53]}$.

综上所述, 通过暖季型和冷季型植物的合理配置可以达到缓解 $\mathrm{C} / \mathrm{N}$ 比失衡湿地秋、冬季碳源不足和提 高系统氮去除率的效果, 暖季型植物在秋、冬季的分解可以向浮床人工湿地系统补充碳源, 提高系统 $\mathrm{C} / \mathrm{N}$ 比, 促进反硝化作用. 但暖季型植物的过量分解会对湿地水体溶解氧浓度及冷季型植物的根系活力产生不 利影响. 因此作为外加碳源的暖季型植物的投加方式和投加量还需进一步研究.

\section{4 参考文献}

[ 1 ] Vymazal J. Horizontal sub-surface flow and hybrid constructed wetlands systems for wastewater treatment. Ecological Engineering, 2005, 25(5) : 478-490.

[ 2 ] Lin YF, Jing SR, Lee DY et al. Nitrate removal from groundwater using constructed wetlands under various hydraulic loading rates. Bioresource Technology, 2008, 99(16): 7504-7513.

[ 3 ] Ruiz AM, Maerz, JC, Davis AK et al. Patterns of development and abnormalities among tadpoles in a constructed wetland receiving treated wastewater. Environmental Science and Technology, 2010, 44(13): 4862-4868.

[ 4 ] Sindilariu PD, Wolter C, Reiter R. Constructed wetlands as a treatment method for effluents from intensive trout farms. Aquaculture, 2008, 277(3): 179-184.

[ 5 ] Vymazal J. Constructed wetlands for treatment of industrial wastewaters: A review. Ecological Engineering, 2014, 73: 724-751.

[ 6 ] Fleming-Singer MS, Horne AJ. Enhanced nitrate removal efficiency in wetland microcosms using an episediment layer for denitrification. Environmental Science and Technology, 2002, 36(6) : 1231-1237.

[ 7 ] Lu SL, Hu HY, Sun YX et al. Effect of carbon source on the denitrification in constructed wetlands. Journal of Environmental Sciences, 2009, 21: 1036-1043.

[ 8 ] Liu Gang, Wen Yue, Zhou Qi. Advance in enhancement of denitrification in the constructed wetlands using external carbon sources. Technology of Water Treatment, 2010, 36(4) : 1-5. [刘刚, 闻岳, 周琪. 人工湿地反硝化碳源补充研究进展. 水处理技术, 2010, 36(4): 1-5.]

[ 9 ] Ding Yi, Song Xinshan, Yan Denghua. The application and research progress of denitrifying carbon source in constructed wetlands for nitrogen removal. Environmental Pollution and Control, 2011, 33(12)：65-70. [丁怡, 宋新山, 严登华. 反 硝化碳源在人工湿地脱氮中的应用及其研究进展. 环境污染与防治, 2011, 33(12): 65-70.]

[10] Wen Y, Chen Y, Zheng N et al. Effects of plant biomass on nitrate removal and transformation of carbon sources in subsurface-flow constructed wetlands. Bioresource Technology, 2010, 101(19) : 7286-7292.

[11] Ingersoll TL, Baker LA. Nitrate removal in wetland microcosms. Water Research, 1998, 32 (3): 667-684.

[12] Wei Xing, Zhu Wei, Zhao Lianfang et al. Effect of the carbon source of plant straw supplement in constructed artificial 
wetland on nitrogen removal. J Lake Sci, 2010, 22(6) : 916-922. DOI:10.18307/2010.0615. [魏星, 朱伟, 赵联芳等. 植物秸秆作补充碳源对人工湿地脱氮效果的影响. 湖泊科学, 2010, 22(6) : 916-922.]

[13] Shao L, Xu ZX, Jin W et al. Rice husk as carbon source and biofilm carrier for water denitrification. Polish Journal of Environmental Studies, 2009, 18(4) : 693-699.

[14] Sun Yali, Zhang Guochen, Yan Zhong et al. Removing nitrate-nitrogen from wastewater using rotten wood as carbon source. Environmental Science, 2010, 31(6): 1494-1498. [孙雅丽, 张国臣, 阎中等. 以腐朽木为碳源去除废水中硝酸盐氮 的研究. 环境科学, 2010, 31(6): 1494-1498.]

[15] Yang Yuting, He Xiaojuan, Su Yuelong et al. Research progress in the enhancement of nitrogen removal using plant biomass as extra carbon source in constructed wetlands. Technology of Water Treatment, 2015, 41(5): 1-4. [杨玉婷, 何小 娟, 苏跃龙等. 外加植物碳源强化人工湿地脱氮的研究进展. 水处理技术, 2015, 41(5): 1-4.]

[16] Xiao Lei, He Feng, Huang Danping et al. Research advances of adding extra carbon sources to denitrification for constructed wetlands. Journal of Hydroecology, 2012, 33(1) : 139-143. [肖蕾, 贺锋, 黄丹萍等. 人工湿地反硝化外加碳源研 究进展. 水生态学杂志, 2012, 33(1): 139-143.]

[17] Shao Liu, Xu Zuxin, Wang Sheng et al. Performance of new solid carbon source materials for denitrification. Environmental Science, 2011, 32(8) : 2323-2327. [邵留, 徐祖信, 王茂等. 新型反硝化固体碳源释碳性能研究. 环境科学, 2011, 32( 8$)$ : 2323-2327.]

[18] Zhong Shengqiang, Yang Yang, Tao Ran et al. Carbon releasing characteristics and denitrification effects of five plant materials. Environmental Engineering, 2014, 8(5):1817-1824. [ 钟胜强, 杨扬, 陶然等. 5 种植物材料的水解释碳性能 及反硝化效率. 环境工程学报, $2014,8(5): 1817-1824$. $]$

[19] Qiang Rongrong, Wang Guoxiang, Zhang Limin et al. The continuous effects of decaying Eichhornia crassipes on water quality. Environmental Monitoring in China, 2005, 21(1): 24-27. [强蓉蓉, 王国祥, 张利民等. 凤眼莲死亡对湖泊 水质的持续性影响分析. 中国环境监测, 2005, 21(1): 24-27.]

[20] Wu Haitao, Lü Xianguo, Yang Qing et al. The early-stage litter decomposition and its influencing factors in the wetland of the Sanjiang Plain, China. Acta Ecologica Sinica, 2007, 27 (10): 4027-4035. [武海涛, 吕宪国, 杨青等. 三江平原典 型湿地枯落物早期分解过程及影响因素. 生态学报, 2007, 27(10): 4027-4035.]

[21] Zhang Zhiliang, Qu Weiqing eds. Plant physiology guide. Beijing: Higher Education Press, 2004. [张志良, 翟伟菁. 植 物生理学实验指导. 北京: 高等教育出版社, 2004.]

[22] Ye Chun, Wang Bo, Li Chunhua et al. Nutrient release process during decomposition of submerged macrophytes (Hydrilla verticillata Royle). Environmental Science, 2014, 34(10): 2653-2659. [叶春, 王博, 李春华等. 沉水植物黑藻腐解过 程中营养盐释放过程. 环境科学, 2014, 34(10): 2653-2659.]

[23] Xie Li, Yang Hao, Qu Xiaoxia et al. Dissolved organic matter release of Zizania caduciflora and Phragmites australis from Lake Dianchi. Environmental Science, 2013, 34(9): 3458-3466. [谢理, 杨浩, 渠晓霞等. 滇池优势挺水植物荠草和 芦苇降解过程中 DOM 释放特征研究. 环境科学, 2013, 34(9) : 3458-3466. ]

[24] Lin Liansheng, Yue Chunmei, Miao Weimin. Hydrilla verticillata and its utilization in aquaculture. Reservoir fisheries, $2005,25(5)$ : 33-34. [ 林连升, 岳春梅, 缪为民. 轮叶黑藻及其在水产养殖上的利用. 水利渔业, 2005, 25(5): 33-34.

[25] Vymazal J. The use of sun-surface constructed wetlands for wastewater treatment in the Czech Republic: 10 years experience. Ecological Engineering, 2002, 18(5) : 633-646.

[26] Tanner CC, Kadlec RH, Gibbs MM et al. Nitrogen processing gradients in subsurface-flow treatment wetlands-influence of wastewater characteristics. Ecological Engineering, 2002, 18(4) : 499-520.

[27] Bulc TG. Long term performance of a constructed wetland for landfill leachate treatment. Ecological Engineering, 2006,26 (4) : 365-374.

[28] Ling Ziwei, Tong Xinnan, Li Yahong et al. Study on nitrogen removal process of treatments for slightly contaminated water on hybrid constructed wetlands. Research of Environmental Sciences, 2013, 26(3):320-325. [凌子微, 全欣楠, 李亚红 等. 处理低污染水的复合人工湿地脱氮过程. 环境科学研究, 2013, 26(3) : 320-325.]

[29] Lee C, Fletcher TD, Sun G. Nitrogen removal in constructed wetland systems. Engineering in Life Sciences, 2009, 9(1): $11-22$.

[30] Lin YF, Jing SR, Wang TW et al. Effects of macrophytes and external carbon sources on nitrate removal from groundwater 
in constructed wetlands. Environmental Pollution, 2002, 119(3) : 413-420.

[31] Peng L, Hua Y, Cai J et al. Effects of plants and temperature on nitrogen removal and microbiology in a pilot-scale integrated vertical-flow wetland treating primary domestic wastewater. Ecological Engineering, 2014, 64(3) : 285-290.

[32] Constructed wetlands and aquatic plant systems for municipal wastewater treatment. US Environmental Protection Agency, Office of Research and Development, 1988.

[33] Fernandez-Nava Y, Maranon E, Soons J et al. Denitrification of high nitrate concentration wastewater using alternative carbon sources. Journal of Hazardous Materials, 2010, 173(1) : 682-688.

[34] Yang Shan, Shi Wenhao, Wang Han et al. Research progress in effect of additional carbon source on water with heterotrophic denitrification. Environmental Science \& Technology, 2014, 37(8): 54-58. [ 杨珊, 石纹豪, 王晗等. 外加碳源 影响水体异养反硝化脱氮的研究进展. 环境科学与技术, 2014, 37(8): 54-58.]

[35] Hume NP, Fleming MS, Home AJ. Denitrification potential and carbon quality of four aquatic plants in wetland microcosms. Soil Science Society of America Journal, 2002, 66(5) : 1706-1712.

[36] Matsumoto S, Terada A, Tsuneda S. Modeling of membrane aerated biofilm: Effects of $\mathrm{C} / \mathrm{N}$ ratio, biofilm thickness and surface loading of oxygen on feasibility of simultaneous nitrification and denitrification. Biochemical Engineering, 2007, 37 (1) : 98-107.

[37] Qian Wei, Lu Kaihong, Zheng Zhongming et al. Effects of carbon source and $\mathrm{C} / \mathrm{N}$ ratio on treatment of aquaculture wastewater by multiple microorganisms. Journal of Fisheries of China, 2012, 36(12) : 1880-1890. [ 钱伟, 陆开宏, 郑忠 明等. 碳源及 $\mathrm{C} / \mathrm{N}$ 对复合菌群净化循环养殖废水的影响. 水产学报, 2012, 36(12): 1880-1890.]

[38] Lu Songliu, Zhang Chen, Wang Guohua. Study on the influence of enhanced carbon resource on denitrification in the constructed wetland. Acta Scientiae Circumstantiae, 2011, 31(9): 1949-1954. [陆松柳, 张辰, 王国华. 碳源强化对人工 湿地反硝化过程的影响研究. 环境科学学报, 2011, 31(9) : 1949-1954.]

[39] Zhao Lianfang, Zhu Wei, Gao Qing. Improving nitrogen removal of constructed wetlands by supplying plant carbon. Journal of Pla University of Science and Technology (Natural Science Edition), 2009, 10(6) : 644-649. [ 赵联芳, 朱伟, 高青. 补充植物碳源提高人工湿地脱氮效率. 解放军理工大学学报 (自然科学版), 2009, 10(6): 644-649.]

[40] Shang Huilai, Peng Yongzhen, Zhang Jingrong et al. The effect of temperature on short-cut nitrification and denitrification. Acta Scientiae Circumstantiae, 2009, 29(3) : 516-520. [尚会来, 彭永榛, 张静蓉等. 温度对短程硝化反硝化的影响. 环境科学学报, 2009, 29(3): 516-520.]

[41] Dai Yanran, Liang Wei, Wu Zhenbin. Research advances of constructed wetlands for nitrogen removal of wastwater with high nitrogen and low carbon contents. Journal of Agro-environment Science, 2010, 29( Suppl) : 305-309. [代嫣然, 梁 威, 吴振斌. 低碳高氮废水的人工湿地脱氮研究进展. 农业环境科学学报, 2010, 29(增刊) : 305-309.]

[42] Zhang Ju, Deng Huanguang, Wang Dongqi et al. Impact of decomposition of submerged macrophytes on nutrient speciation concentration in overlying water of Tuhai River. Environmental Science \& Technology, 2011, 34(5) : 14-19. [张菊, 邓焕 广, 王东启等. 徒骇河沉水植物腐烂对上覆水体中营养盐形态变化影响. 环境科学与技术, 2011, 34(5) : 14-19.]

[43] Chen Y, Wen Y, Cheng J et al. Effects of dissolved oxygen on extracellular enzymes activities and transformation of carbon sources from plant biomass: Implications for denitrification in constructed wetlands. Bioresource Technology, 2011, 102 (3) : 2433-2440.

[44] Bastviken SK, Eriksson PG, Premrov A et al. Potential denitrification in wetland sediments with different plant species detritus. Ecological Engineering, 2005, 25(2) : 183-190.

[45] Chen Y, Wen Y, Zhou Q et al. Effects of plant biomass on denitrifying genes in subsurface-flow constructed wetlands. Bioresource Technology, 2014, 157(2) : 341-345.

[46] Faulwetter JL, Gagnon V, Sundberg C et al. Microbial processes influencing performance of treatment wetlands: A review. Ecological Engineering, 2009, 35(6): 987-1004.

[47] Wang Danying, Han Bo, Zhang Xiufu et al. Influence of rhizosphere oxygen concentration on rice root growth. Acta Agronomica Sinica, 2008, 34(5): 803-808. [王丹英, 韩勃, 章秀福等. 水稻根际含氧量对根系生长的影响. 作物学报, $2008, \mathbf{3 4}(5): 803-808$. ]

[48] Xu Chunmei, Wang Danying, Chen Song et al. Effect of aeration on root growth and nitrogen metabolism in rice. Chinese Journal of Rice Science, 2012, 26(3) : 320-324. [徐春梅, 王丹英, 陈松等. 增氧对水稻根系生长与氮代谢的影响. 中国水稻科学, 2012, 26(3): 320-324.] 
[49] Zou X, Zhang H, Zuo J et al. Decreasing but still significant facilitation effect of cold-season macrophytes on wetlands purification function during cold winter. Scientific Reports, 2016, 6: 27011.

[50] Meng P, Pei H, Hu W et al. How to increase microbial degradation in constructed wetlands: Influencing factors and improvement measures. Bioresource Technology, 2014, 157(4) : 316-326.

[51] Wang Shengrui, Nian Yuegang, Hou Wenhua et al. Macrophyte selection in artificial wetlands. J Lake Sci, 2004, 16 (1) : 91-96. DOI: 10.18307/2004.0115. [王圣瑞, 年跃刚, 侯文华等. 人工湿地植物的选择. 湖泊科学, 2004, 16(1): 91-96. ]

[52] Chen Mingli, Wu Xiaofu, Chen Yonghua et al. Construction of a landscaping-type wetland system for wastewater treatment and analysis of plant denitrifying effect. Environmental Science, 2010, 31(3): 660-666. [陈明利, 吴晓芙, 陈永华等. 景观型人工湿地污水处理系统构建及植物脱氮效应研究. 环境科学, 2010, 31(3): 660-666.]

[53] Du Xiaoli, Xu Zuxin, Zheng Lei. Study on atmospheric oxygenation in simulate vertical-flow constructed wetland. Environmental Pollution and Control, 2013, 35(5): 31-34. [杜晓丽, 徐祖信, 郑否. 模拟垂直潜流人工湿地大气复氧规律 研究. 环境污染与防治, 2013, 35(5): 31-34.] 